\title{
Expression of LRRC8A is elevated in the cytoplasm of osteosarcoma tissues: An immunohistochemical study with tissue microarrays
}

\author{
NINGFENG ZHANG* ${ }^{*}$ ZHIQIN DENG* ${ }^{*}$ WENCUI LI, YAN ZOU, JIANYI XIONG, LI DUAN and DAPING WANG \\ Key Laboratory of Tissue Engineering, Shenzhen Second People's Hospital, \\ The First Affiliated Hospital of Shenzhen University, Shenzhen, Guangdong 518000, P.R. China
}

Received May 5, 2020; Accepted October 28, 2020

DOI: $10.3892 /$ etm.2020.9503

\begin{abstract}
The purpose of the present study was to investigate the expression profile of leucine-rich repeat-containing protein 8A (LRRC8A) in osteosarcoma and normal cortical bone, as well as its association with sex, age and tumor malignancy. Immunohistochemical staining of osteosarcoma tissue microarrays (TMAs) was performed to determine the protein expression of LRRC8A and compare them among different subgroups. The expression of LRRC8A in the nuclei and cytoplasm of U2OS tumor cells and MC3T3-E1 osteoblast-like cells was determined using reverse transcription-quantitative PCR. Of all samples of the TMA for patients with osteosarcoma that were tested, 94\% featured high cytoplasmic expression of LRRC8A, while in all normal bone tissue control groups, the gene was mainly expressed in the nucleus. In MC3T3-E1 osteoblasts, the expression of LRRC8A at the RNA level was mainly in the cytoplasm. The difference in expression of LRRC8A between microarrays and osteoblasts was statistically significant. In U2OS osteosarcoma cells, LRRC8A mRNA was concentrated in the nuclei and cytoplasm. In osteosarcoma, the expression level of LRRC8A was not significantly associated with sex or age. In conclusion, LRRC8A was highly expressed in the cytoplasm of osteosarcoma cells and the degree of expression may be associated with the degree of tumor malignancy.
\end{abstract}

Correspondence to: Dr Li Duan or Dr Daping Wang, Key Laboratory of Tissue Engineering, Shenzhen Second People's Hospital, The First Affiliated Hospital of Shenzhen University, 3002 SunGang West Road, Shenzhen, Guangdong 518000, P.R. China E-mail: 825343512@qq.com

E-mail: dapingwang1963@qq.com

*Contributed equally

Key words: osteosarcoma, leucine-rich repeat-containing protein $8 \mathrm{~A}$, volume-regulated anion channels, tissue microarray, immunohistochemistry

\section{Introduction}

Osteosarcoma is a common type of primary malignant bone tumor (1), that occurs mostly in adolescents (2). Highly invasive, it easily invades the long-growing metaphysis (3) and it most commonly metastasizes to bone and the lungs (4). The high mortality rate of osteosarcoma is related to systemic metastasis (5). Despite the multimodal combination of chemotherapy and extensive tumor resection, the 5-year survival rate is only $60-70 \%(2,6)$. Therefore, osteosarcoma requires further study. Gene therapy may be a potential mode of osteosarcoma treatment (7).

Cell volume regulation has an important role in various cellular functions, such as cell metabolism, proliferation, migration and death $(8,9)$. Channel proteins on the cell membrane regulate the cell volume by controlling the movement of water and electrolytes. Although channel proteins have been indicated to have important roles in a variety of physiological processes, the molecular mechanisms of volume-regulated anion channels (VRACs) remain to be fully elucidated (10). Leucine-rich repeat-containing 8A (LRRC8A) is a major molecular determinant of the VRAC current, which has been proven by previous studies $(11,12)$.

Although the primary function of VRACs is cell volume regulation, they are considered potential targets for cancer therapy in view of their important roles in cell proliferation, migration and apoptosis of both normal and cancer cells (13-15). LRRC8A has been isolated from and identified in patients with congenital gammaglobulinemia (16). It was reported to be involved in inflammation by supporting TNF- $\alpha$-induced superoxide production in vascular smooth-muscle cells (17). In glioblastoma, downregulation of LRRC8A inhibits proliferation and increases sensitivity to temozolomide and carmustine (18). In colon cancer, LRRC8A was determined to be highly expressed and it was indicated to promote the growth and metastasis of cancer cells (19). In ovarian cancer and alveolar-cell carcinoma, a decrease in LRRC8A may be a factor affecting cisplatin resistance $(20,21)$. However, the expression of LRRC8A in osteosarcoma has remained elusive. Therefore, in the present study, the expression profile of LRRC8A in osteosarcoma was determined using tissue microarrays (TMAs) and immunohistochemistry (IHC). 
The expression of LRRC8A in the nuclei and cytoplasm of U2OS tumor cells and MC3T3-E1 osteoblast-like cells was determined using reverse transcription-quantitative (RT-q) PCR.

\section{Materials and methods}

TMAs and pathology. Paraffin-embedded tumor TMAs (cat. no. BO481a) were purchased from US Biomax, Inc. Specimens on the TMAs were from 40 patients (18 with osteosarcoma, 19 with chondrosarcoma and 3 with giant-cell tumors of the bone) and 8 healthy subjects ( 6 of marginal bone and bone marrow tissue, 2 of cartilage tissue). Osteosarcoma grades IA-IIB and chondrosarcoma were classified into lowly differentiated, moderately differentiated and well-differentiated tissues. There were a total of 96 tissue samples on the microarray, 2 from each patient.

IHC. TMAs were routinely dewaxed with xylene and hydrated with an alcohol gradient. They were then subjected to antigen retrieval using a $0.01 \mathrm{M}$ sodium citrate buffer $(\mathrm{pH} 6.0$, $95^{\circ} \mathrm{C}$ for $\left.15 \mathrm{~min}\right)$. Endogenous peroxidase was blocked with $3 \% \mathrm{H}_{2} \mathrm{O}_{2}$-methanol for $10 \mathrm{~min}$ at room temperature. The TMAs were then incubated with normal goat serum (UltraSensitive $^{\text {TM }}$ SP; KIT-9720; MAXIM) for $10 \mathrm{~min}$ at room temperature to block non-specific binding. Subsequently, samples were incubated overnight with ready-to-use primary antibody (UltraSensitive ${ }^{\mathrm{TM}}$ SP; KIT-9720; MAXIM) at $4^{\circ} \mathrm{C}$ according to manufacturer's method. After three washes with PBS, the TMAs were incubated with biotinylated goat anti-mouse/rabbit immunoglobulin G secondary antibody (UltraSensitive $^{\mathrm{TM}}$ SP; KIT-9720; MAXIM) for $10 \mathrm{~min}$ at room temperature, according to manufacturer's method. Next, the TMAs were incubated with streptavidin-peroxidase for $10 \mathrm{~min}$ at room temperature. Finally, blots were visualized using diaminobenzidine (DAB)/ACE (DAB color development kit; MAXIM) for 10 min and the development was stopped by adding distilled water (according to manufacturer's method).

Imaging and data analysis. IHC staining images were acquired using an Olympus BX60 microscope (Olympus Corp.) equipped with a Leica DP70 digital camera (Leica Microsystems). A pathologist (double-blinded) scored staining using the previously described four-point system (score 0-3) (22) as follows: Score 3, dark staining that was easily visible and present in $>50 \%$ of cells; score 2 , focal areas of dark staining ( $<50 \%$ of cells) or moderate staining in $>50 \%$ of cells; score 1 , focal moderate staining in $<50 \%$ of cells, or pale staining in any proportion of cells not easily observable at low power; and score 0 , none of the above. A high level of expression was defined as a score of 2-3 and a low level as a score of $0-1$, as described previously.

Cell culture. The osteosarcoma cell line U2OS (Cell Bank of the Chinese Academy of Sciences) and the osteoblast-like cell line ME3T3-E1 (Cell Center, Institute of Basic Medicine, Chinese Academy of Medical Sciences) were cultured in DMEM (Gibco; Thermo Fisher Scientific, Inc.) containing 10\% fetal bovine serum (Gibco; Thermo Fisher Scientific, Inc.) and double antibodies (1\% penicillin and streptomycin).
The medium was changed every 2-3 days and the culture conditions in a humidified atmosphere $\left(37^{\circ} \mathrm{C}\right.$ and $\left.5 \% \mathrm{CO}_{2}\right)$. When the cell confluence reached $80-90 \%$, the cells were used for the experiments.

RT-qPCR. A Cytoplasmic and Nuclear Ribonucleic Acid (RNA Purification Kit; (Norgen Biotek Corp.) was used to separate and extract cytoplasmic and nuclear RNA from U2OS and MC3T3-E1 cells. They were immediately reverse-transcribed to complementary (c)DNA using an PrimeScript ${ }^{\mathrm{TM}}$ RT reagent kit (cat. no. RR037A; Takara Bio, Inc.), according to the manufacturer's protocol. The amplification reaction conditions were $42^{\circ} \mathrm{C}$ for $2 \mathrm{~min}$ and a hold at $4^{\circ} \mathrm{C}$. RT conditions were $37^{\circ} \mathrm{C}$ for $15 \mathrm{~min}, 85^{\circ} \mathrm{C}$ for $5 \mathrm{sec}$ and a hold at $4^{\circ} \mathrm{C}$. Subsequently, qPCR was performed by using the kit (PrimeScript ${ }^{\mathrm{TM}}$ RT reagent kit; cat. no. RR037A; Takara Bio, Inc.) on the extracted cDNA, according to the manufacturer's protocols. SYBR ${ }^{\circledR}$ Premix Ex Taq ${ }^{\mathrm{TM}}$ II (cat. no. RR820A; Takara Bio, Inc.) was used to perform qPCR. The reaction conditions were as follows: Step $1,95^{\circ} \mathrm{C}$ for $10 \mathrm{sec}$; Step $2,95^{\circ} \mathrm{C}$ for $5 \mathrm{sec}$ and $60^{\circ} \mathrm{C}$ for $30 \mathrm{sec}$, the cycle was repeated for 40 times; and Step $3,95^{\circ} \mathrm{C}$ for $15 \mathrm{sec}, 60^{\circ} \mathrm{C}$ for $60 \mathrm{sec}$ and $95^{\circ} \mathrm{C}$ for $15 \mathrm{sec}$. GAPDH was used as the internal reference gene. The primers were as follows: GAPDH forward, 5'-GGCACAGTCAAG GCTGAGAATG-3' and reverse, 5'-ATGGTGGTGAAGACG CCAGTA-3'; LRRC8A forward, 5'-TCACAGCCAATAGGA TTGAAGC-3' and reverse, 5'-CCTAGCCCAGTGCCAATA AG-3'. Ribozyme-free operation was ensured throughout the process. The $2^{-\Delta \Delta \mathrm{Cq}}$ method was used to determine the relative expression levels of LRRC8A (23).

Statistical analysis. Data were compared between groups using a $\chi^{2}$ or Student's t-test. SPSS software version 21.0 (IBM Corp.) was used for all analyses. All P-values were two-tailed and $\mathrm{P}<0.05$ was considered to indicate statistical significance.

\section{Results}

Subcellular localization of LRRC8A. IHC analysis suggested that LRRC8A was present in the nuclei and cytoplasm of osteosarcoma and giant-cell tumor cells (Fig. 1A-D). In normal bone tissues, it was mainly expressed in the nucleus (Figs. 1G and 2). In MC3T3-E1 osteoblasts, the expression of LRRC8A at the RNA level was mainly in the cytoplasm and the difference was statistically significant (Fig. 2). In U2OS osteosarcoma cells, the expression of LRRC8A at the RNA level was expressed in the nuclei and cytoplasm and the difference was not statistically significant (Fig. 3). The expression level of LRRC8A was relatively low in chondrosarcoma and cartilage tissues. However, the expression was slightly higher in poorly differentiated chondrosarcoma (Fig. 1E, F and H).

Expression of LRRC8A in the cytoplasm of bone tumors. Levels of LRRC8A immunoreactivity in the cytoplasm were compared between normal tissues and bone tumors. 18 osteosarcoma tissue samples on the TMA, 94\% (17/18) exhibited high levels of LRRC8A expression in the cytoplasm (score 2-3; Fig. 1A-C), whereas six bone tissue samples did not. The cytoplasm of osteosarcoma samples had significantly higher 
A

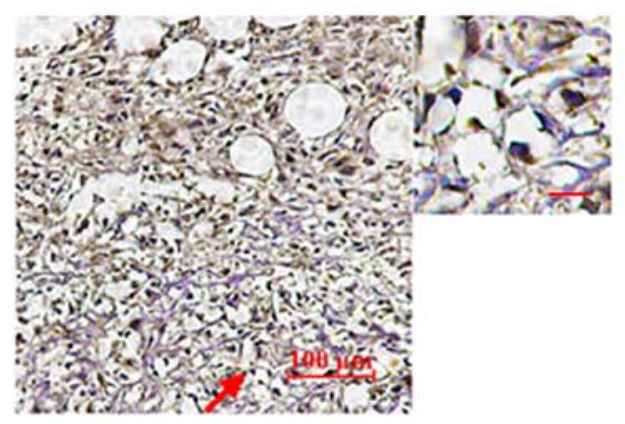

C

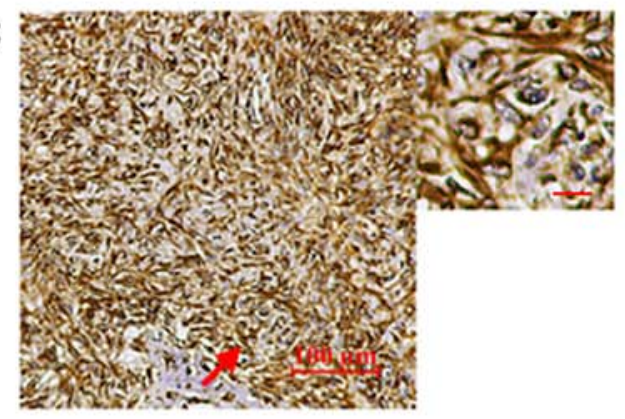

E

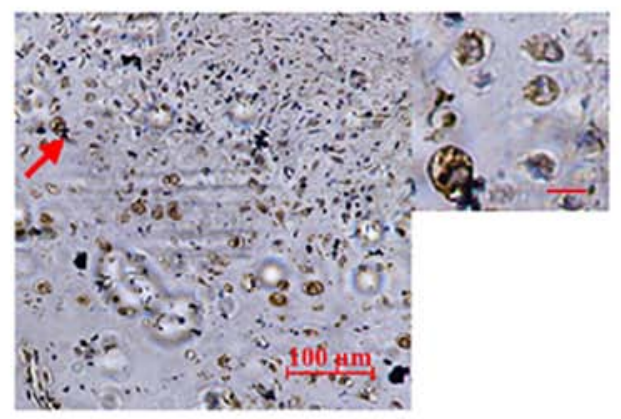

G

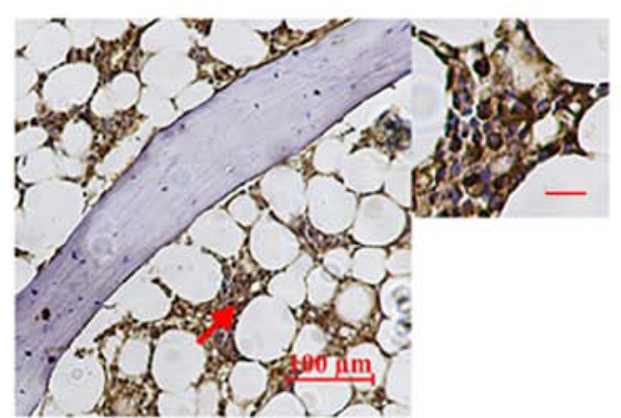

B

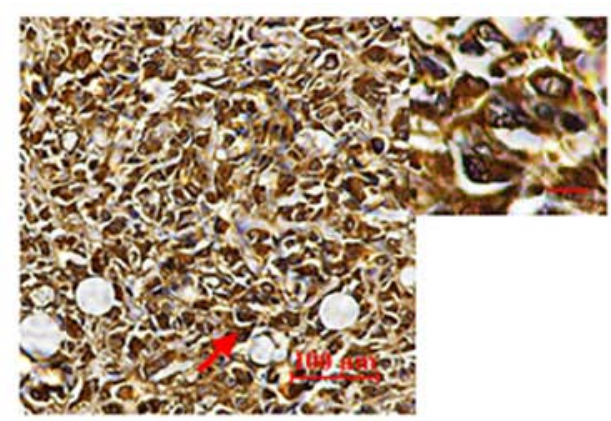

D

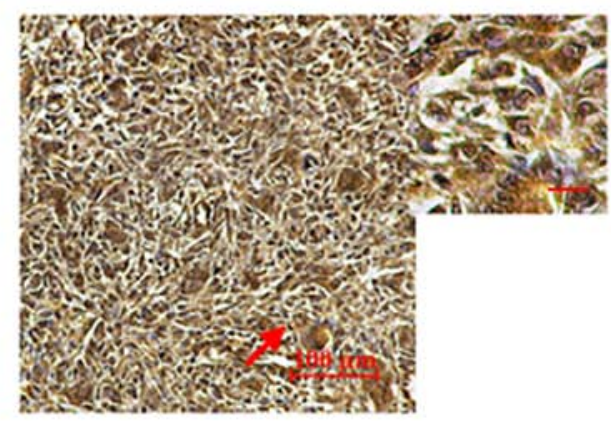

F

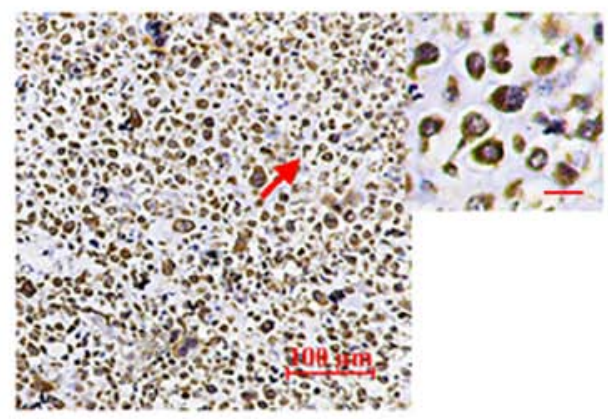

$\mathrm{H}$

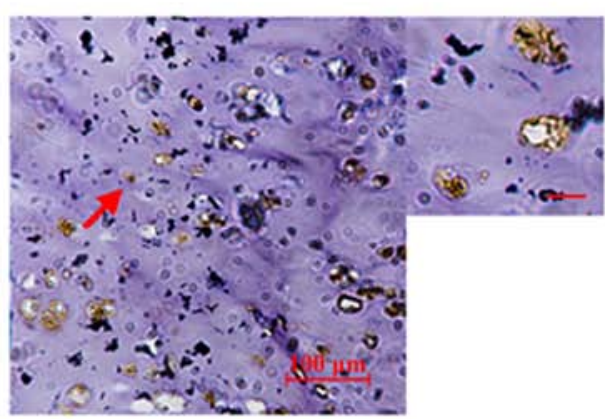

Figure 1. Representative immunohistochemical staining for LRRC8A in normal and bone tumors. Enlarged windows (scale bar, $20 \mu \mathrm{m}$ ) of the areas indicated by arrows are presented in the upper-right corner of each subfigure. LRRC8A was (A) highly expressed in the cytoplasm in stage IA osteoblastic osteosarcoma; (B) highly expressed in the cytoplasm in stage IIB osteoblastic osteosarcoma; (C) highly expressed in the cytoplasm in stage IIB fibroblastic osteosarcoma; and (D) highly expressed in the cytoplasm in grade II giant-cell tumors of the bone. (E) Well-differentiated chondrosarcoma. (F) Poorly differentiated chondrosarcoma. (G) In normal bone tissue, LRRC8A was highly expressed in the nucleus, while its expression in the cytoplasm was low. (H) Normal cartilage tissue (scale bars, $100 \mu \mathrm{m}$ ). LRRC8A, leucine-rich repeat-containing protein 8A.

levels of LRRC8A than that of normal bone tissues $(\mathrm{P}<0.001$; Fig. 4A). Of the 19 chondrosarcoma samples, 16\% (3/19) had high levels of LRRC8A (Fig. 4B); overall, the expression of LRRC8A in chondrosarcoma tissue did not significantly differ from normal cartilage tissue ( $\mathrm{P}>0.05$; Fig. 4B). Osteosarcoma samples had high levels of LRRC8A in the cytoplasm, differing significantly from chondrosarcoma samples with this regard
$(\mathrm{P}<0.001$; Fig. $4 \mathrm{C})$. Compared with those of normal bone tissue, the cytoplasmic LRRC8A levels of giant-cell tumors of the bone were significantly increase $(\mathrm{P}<0.05$; Fig. 4D). In addition, although the number of cases with tumor staging was insufficient, it may be observed from Fig. 1 that a higher grade of osteosarcoma was paralleled by a higher cytoplasmic expression level of LRRC8A. 

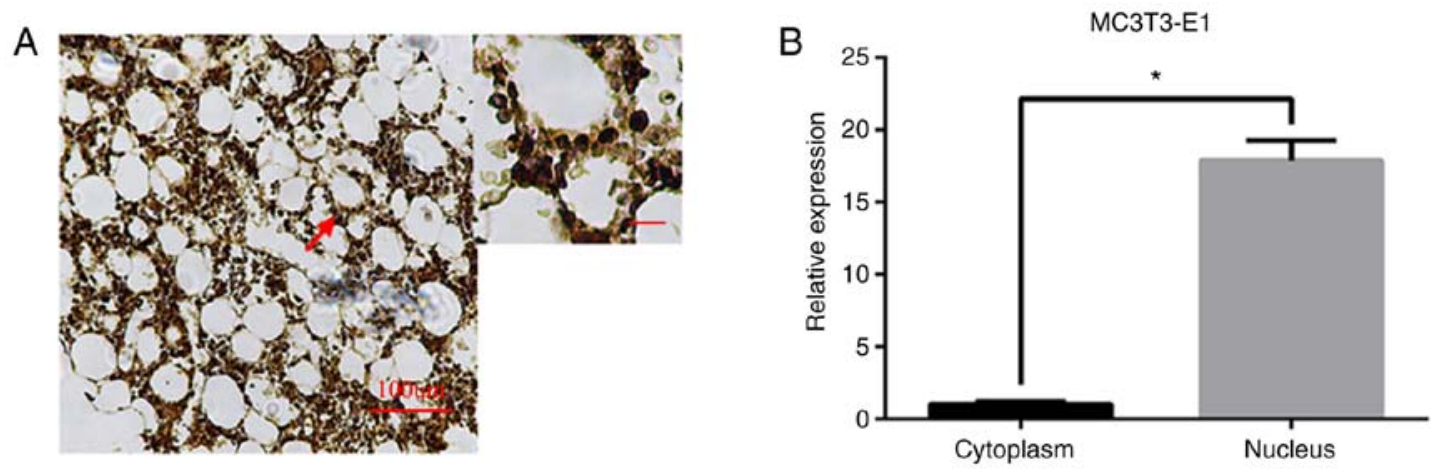

Figure 2. (A) Representative immunohistochemical staining of tissue microarrays was used to detect LRRC8A expression in normal bone tissue (scale bar, $100 \mu \mathrm{m}$ ). An enlarged window (scale bar, $20 \mu \mathrm{m}$ ) of the area indicated by the arrow is presented in the upper-right corner. (B) Reverse transcription-quantitative PCR was performed to detect LRRC8A expression at the RNA level in the nuclei and cytoplasm of MC3T3-E1 osteoblast-like cells. In MC3T3-E1 osteoblast-like cells, LRRC8A is mainly expressed in the nucleus. " $\mathrm{P}<0.05$. LRRC8A, leucine-rich repeat-containing protein $8 \mathrm{~A}$.

A

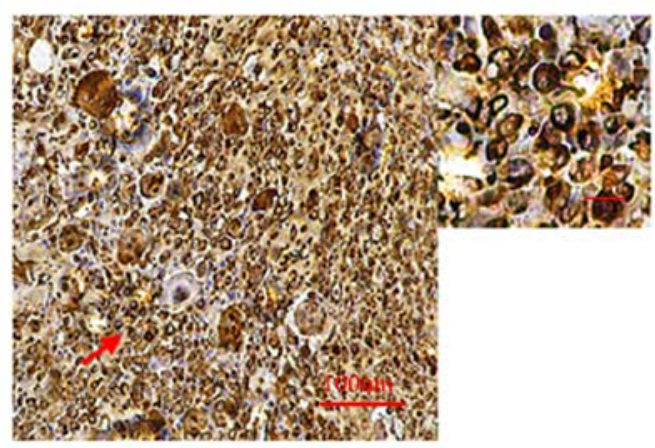

B

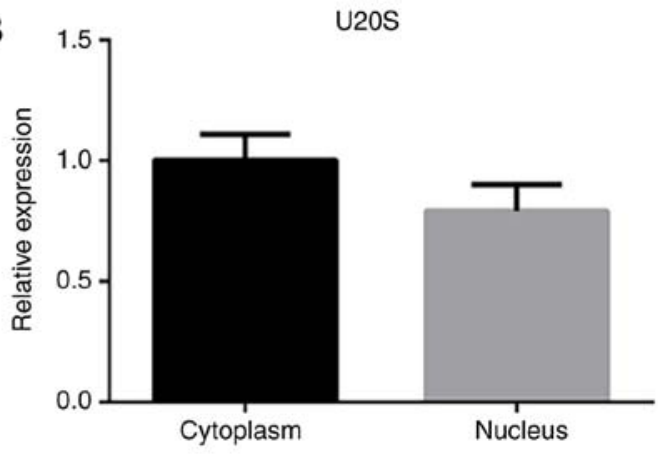

Figure 3. (A) Immunohistochemical staining of tissue microarrays was used to detect LRRC8A expression in osteosarcoma tissue (scale bar, $100 \mu \mathrm{m}$ ). An enlarged window (scale bar, $20 \mu \mathrm{m}$ ) of the area indicated by the arrow is presented in the upper-right corner. (B) Expression of LRRC8A in the nuclei and cytoplasm of U2OS cells was detected using reverse transcription-quantitative PCR. Compared with MC3T3-E1 osteoblast-like cells, there is no significant difference in the expression of LRRC8A in the cytoplasm and nucleus of osteosarcoma cells. LRRC8A, leucine-rich repeat-containing protein 8A.

Sex-associated differences in LRRC8A in bone tumors. The occurrence of bone tumors is sex-dependent; osteosarcoma and chondrosarcoma occur more frequently in males than in females $(24,25)$. Therefore, in the present study, the expression of LRRC8A was compared between bone tumors from males and those from females. Of the 40 patients with bone tumors, samples with high cytoplasmic LRRC8A levels accounted for 61\% (14/23) of males and $53 \%(9 / 17)$ of females and there was no significant difference between sexes ( $\mathrm{P}>0.05$; Fig. $5 \mathrm{~A})$. Of the 18 patients with osteosarcoma, only 1 (a male) had low expression of LRRC8A, while the remainder (9 males and 8 females) had high expression of LRRC8A, with no sex-related difference ( $\mathrm{P}>0.05$; Fig. 5B). Of the 19 patients with chondrosarcoma, only 3 males had high expression of LRRC8A and the other 16 patients ( 8 males, 8 females) had low expression thereof. Therefore, no sex-related differences in LRRC8A expression were detected ( $\mathrm{P}>0.05$; Fig. 5C).

Age-associated differences in LRRC8A in bone tumors. As the incidence of bone tumors also differs by age (26); the effect of age on LRRC8A immunoreactivity in bone tumor tissues was also investigated. Among all bone tumor patients, the average age was 32.4 years. The percentage of samples with high cytoplasmic expression of LRRC8A was 33\% (6/18) among those from patients aged $\geq 32.4$ years and $77 \%$ (17/22) among those from patients aged $<32.4$ years. Statistical analysis indicated a significant difference between these two age groups $(\mathrm{P}<0.05$; Fig. 6A). Among the 18 patients with osteosarcoma, the average age was 27.1 years. In patients aged $\geq 27$ years, $100 \%$ had high expression of LRRC8A and in the group aged $<27$ years, $89 \%$ had high expression of LRRC8A. Statistical analysis indicated no significant difference between these two age groups $(\mathrm{P}>0.05$; Fig. 6B). Among the 19 cases of chondrosarcoma, the average patient age was 37.5 years. Only one patient aged $\geq 37.5$ years and two patients aged $<37.5$ years had high levels of LRRC8A and statistical analysis indicated no significant difference between these two age groups ( $\mathrm{P}>0.05$; Fig. 6C).

\section{Discussion}

The results of the TMA of the present study indicated that LRRC8A was expressed in the cytoplasm and nuclei of osteosarcoma cells, while it was mainly expressed in the nuclei of normal bone tissue cells. To verify this phenomenon, RT-qPCR was used to analyze the expression of LRRC8A in the nuclei and cytoplasm of U2OS osteosarcoma cells and MC3T3-E1 osteoblast-like cells and the results were consistent with the IHC results. Analysis of the IHC images suggested that a higher degree of malignancy in osteosarcoma was 

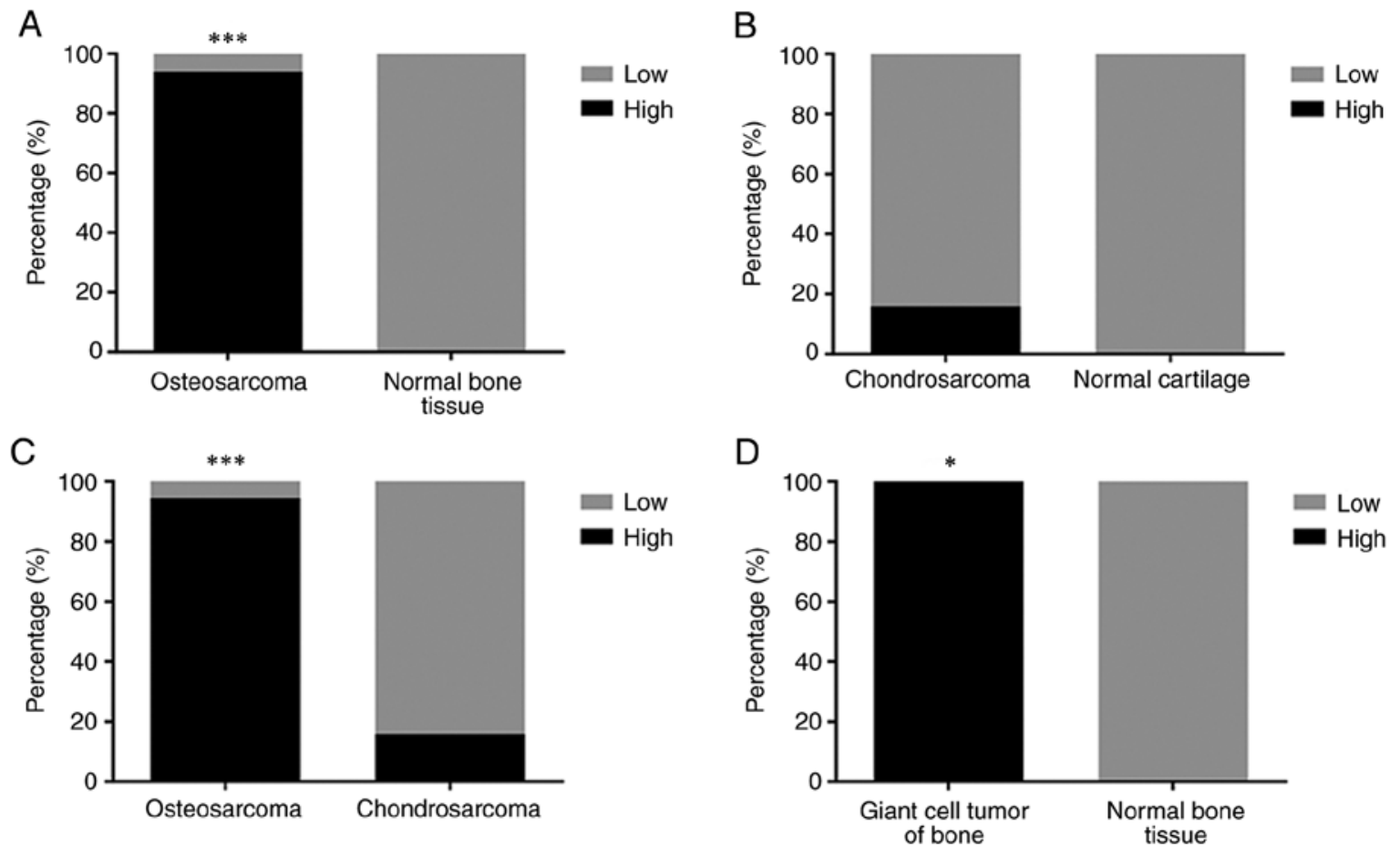

Figure 4. Statistical analysis of the LRRC8A immune response in normal bone and bone tumors. (A) Percentage of samples with high cytoplasmic LRRC8A levels in normal bone tissue vs. osteosarcoma tissues. (B) Percentage of samples with high LRRC8A levels in normal cartilage tissue vs. chondrosarcoma tissue. (C) Percentage of samples with high LRRC8A levels in osteosarcoma tissue vs. chondrosarcoma tissue. (D) Percentage of samples with high cytoplasmic LRRC8A levels in normal bone tissue vs. giant-cell tumor of bone. ${ }^{*} \mathrm{P}<0.05 ;{ }^{* * *} \mathrm{P}<0.001$ vs. normal; $\chi^{2}$ or Fisher's exact test. LRRC8A, leucine-rich repeat-containing protein $8 \mathrm{~A}$.
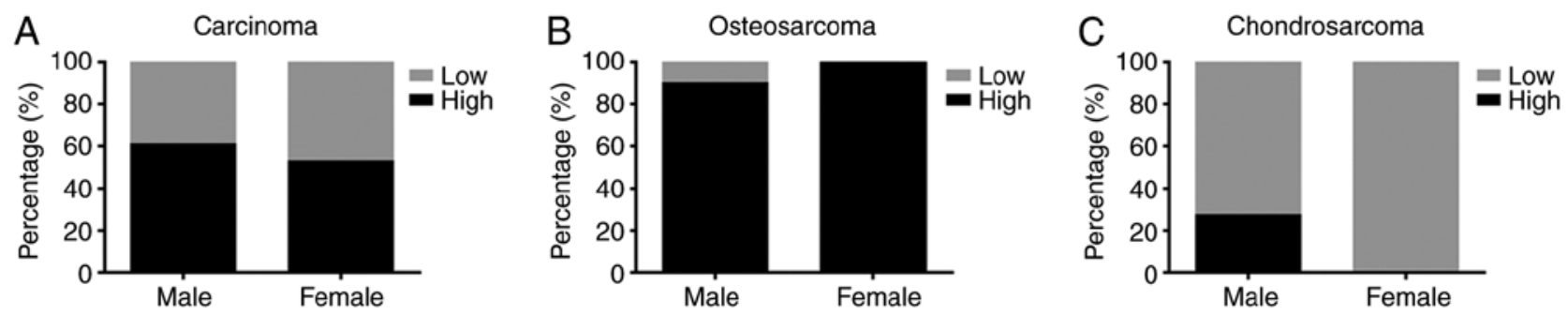

Figure 5. Sex-related analysis of immunoreactivity of LRRC8A in cytoplasm of bone tumors. Comparison of the distribution of samples with high and low expression of LRRC8A between sexes in (A) bone tumors, (B) osteosarcoma tissues and (C) chondrosarcoma tissues. No significant sex-related differences were detected. LRRC8A, leucine-rich repeat-containing protein 8A.
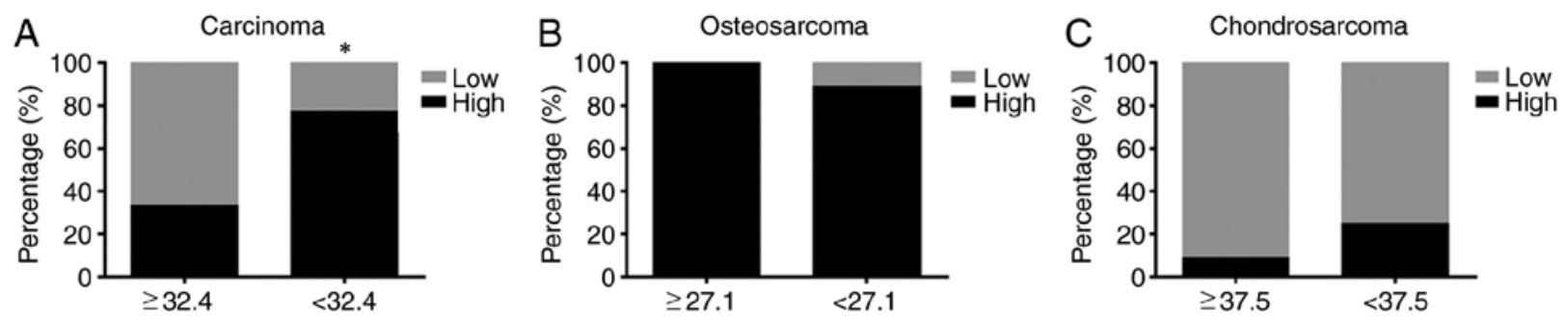

Figure 6. Age-dependent analysis of immunoreactivity of LRRC8A in cytoplasm of bone tumors. Comparison of the distribution of samples with high and low expression of LRRC8A between different age groups in (A) bone tumors, (B) osteosarcoma tissues and (C) chondrosarcoma tissues. The average age in the respective group was used as the cutoff. Ages are presented in years. Among all bone tumor samples tested, LRRC8A expression was higher in patients aged $<32.4$ years. "P $<0.05 ; \chi^{2}$ or Fisher's exact test. No age-related differences were detected in osteosarcoma or chondrosarcoma tissue. LRRC8A, leucine-rich repeat-containing protein $8 \mathrm{~A}$.

paralleled by a higher expression of LRRC8A; furthermore, a lower degree of differentiation in chondrosarcoma was also associated with higher expression of LRRC8A (due to the low osteosarcoma grade). The present results also suggested that in osteosarcoma, the expression of LRRC8A was independent of sex and age. However, in all bone tumor samples, younger 
patients exhibited higher levels of LRRC8A expression $(\mathrm{P}<0.05)$. The results suggested that LRRC8A was increased in the cytoplasm of osteosarcoma cells as compared with that of normal bone tissue. The expression of LRRC8A in bone tumor cells was preliminarily verified at the RNA and protein levels. It was previously reported that LRRC8A is mainly expressed on the cell membrane of colon cancer cells (19).

The limitations of the present study were as follows: The expression of LRRC8A in osteosarcoma and normal bone tissue at the RNA level was studied using U2OS osteosarcoma cells and MC3T3-E1 osteoblast-like cell lines. LRRC8A expression was did not quantitatively analyzed at the protein level in either osteosarcoma or bone cells. In addition, the expression of LRRC8A was not quantified in tissues from patients and healthy volunteers and the use of 2 different cell lines is not sufficient. The subcellular expression and functions of LRRC8A in osteosarcoma require further study. For instance, the whole-cell patch-clamp technique may be used to record the current difference between osteosarcoma cells and normal bone cells (19). After blocking the LRRC8A chloride channel with small interfering (si)RNA or 4-(2-Butyl-6,7-dichloro-2-cyclopentylindan-1-on-5-yl) oxybutyric acid inhibitor, the difference in the change of the chloride current may be observed in order to investigate the function of the LRRC8A chloride channel in osteosarcoma. In recent years, the role of LRRC8A in cancer has been researched. Knockdown of LRRC8A in glioblastoma reduced cell proliferation and increased the sensitivity of the cells to temozolomide and carmustine (18). After knockout of LRRC8A in HCT116 colon cancer cells, the chloride current and cell migration were significantly inhibited and the incidence of tumors in nude mice was also significantly reduced (19). In cisplatin-insensitive cells, transient downregulation of LRRC8A reduced p53 activation and contributed to cisplatin resistance in ovarian and lung cancer cells (20). A study by Konishi et al (27) suggested that LRRC8A is of great significance to the proliferation, survival and migration of esophageal squamous-cell carcinoma (ESCC) cell lines. High expression of LRRC8A was determined to be an indicator of poor prognosis in ESCC (27). Lu et al (28) indicated that in the process of cerebrovascular remodeling induced by angiotensin II, the expression of LRRC8A in human-brain vascular smooth-muscle cells (HBVSMCs) increases. In addition, siRNA-mediated knockout of LRRC8A significantly inhibited the proliferation, migration and invasion of HBVSMCs (28).

The above studies pointed out that high expression of LRRC8A increases cell proliferation, migration and invasion, whether in normal or tumor cells. However, completely reducing the expression of LRRC8A in the cells of animals may negatively affect the proliferation and migration of normal cells. For instance, LRRC8A has an important role in the development and function of T cells $(29,30)$. This said, the location, roles and mechanisms of LRRC8A in cells remain to be fully elucidated. Therefore, the subcellular distribution of LRRC8A in cells and the functions of different parts require to be further studied. Whether the subcellular distribution and physiological functions of LRRC8A differ between normal rapidly proliferating cells (such as stem cells) and tumor cells also requires further study.

In conclusion, the present study suggested that LRRC8A was more highly expressed in the cytoplasm of osteosarcoma cells than in that of normal bone cells. The expression was also associated with the degree of osteosarcoma malignancy. The subcellular locations and physiological functions of LRRC8A in normal rapidly proliferating cells (such as stem cells) and in tumor cells require further study.

\section{Acknowledgements}

Not applicable.

\section{Funding}

This work was supported by the Natural Science Foundation of China (grant nos. 81800785, 81572198 and 81772394), Shenzhen Peacock Project (grant no. KQTD20170331100838136), Shenzhen Science and Technology Projects (grant nos. JCYJ20170817172023838, JCYJ20170306092215436, JCYJ20170412150609690，JCYJ20170413161649437, JCYJ20170413161800287，SGLH20161209105517753, JCYJ20160301111338144 and JCYJ20150330102720175) and the Fund for High-Level Medical Discipline Construction of Shenzhen University (grant no. 2016031638).

\section{Availability of data and materials}

The datasets used and/or analyzed during the current study are available from the corresponding author on reasonable request.

\section{Authors' contributions}

$\mathrm{NZ}$ and ZD performed the experiments, collected the results and wrote the manuscript. WL, YZ and JX contributed to data analysis and manuscript revision. LD and DW conceived the study and contributed to reviewing/editing the manuscript. All authors read and approved the final manuscript.

\section{Ethics approval and consent to participate}

This study was approved by Shenzhen Second People's Hospital (Shenzhen, China).

\section{Patient consent for publication}

Not applicable.

\section{Competing interests}

The authors declare that they have no competing interests.

\section{References}

1. Heare T, Hensley MA and Dell'orfano S: Bone tumors: Osteosarcoma and Ewing's sarcoma. Curr Opin Pediatr 21: 365-372, 2009.

2. Mirabello L, Troisi RJ and Savage SA: International osteosarcoma incidence patterns in children and adolescents, middle ages and elderly persons. Int J Cancer 125: 229-234, 2009.

3. Eppert K, Wunder JS, Aneliunas V, Kandel R and Andrulis IL: von Willebrand factor expression in osteosarcoma metastasis. Mod Pathol 18: 388-397, 2005.

4. Laverdiere C, Hoang BH, Yang R, Sowers R, Qin J, Meyers PA, Huvos AG, Healey JH and Gorlick R: Messenger RNA expression levels of CXCR4 correlate with metastatic behavior and outcome in patients with osteosarcoma. Clin Cancer Res 11: 2561-2567, 2005. 
5. Urakawa H, Nishida Y, Nakashima H, Shimoyama Y, Nakamura $\mathrm{S}$ and Ishiguro $\mathrm{N}$ : Prognostic value of indoleamine 2,3-dioxygenase expression in high grade osteosarcoma. Clin Exp Metastasis 26: 1005-1012, 2009.

6. Bacci G, Ferrari S, Tienghi A, Bertoni F, Mercuri M, Longhi A, Fiorentini G, Forni C, Bacchini P, Rimondini S, De Giorgi U and Picci P: A comparison of methods of loco-regional chemotherapy combined with systemic chemotherapy as neo-adjuvant treatment of osteosarcoma of the extremity. Eur J Surg Oncol 27: 98-104, 2001.

7. Tan ML, Choong PF and Dass CR: Osteosarcoma: Conventional treatment vs. gene therapy. Cancer Biol Ther 8: 106-117, 2009.

8. Lang F, Busch GL, Ritter M, Völkl H, Waldegger S, Gulbins E and Häussinger D: Functional significance of cell volume regulatory mechanisms. Physiol Rev 78: 247-306, 1998.

9. Pedersen SF, Hoffmann EK and Novak I: Cell volume regulation in epithelial physiology and cancer. Front Physiol 4: 233, 2013.

10. Pedersen SF, Klausen TK and Nilius B: The identification of a volume-regulated anion channel: An amazing Odyssey. Acta Physiol (Oxf) 213: 868-881, 2015.

11. Syeda R, Qiu Z, Dubin AE, Murthy SE, Florendo MN, Mason DE, Mathur J, Cahalan SM, Peters EC, Montal M and Patapoutian A: LRRC8 proteins form volume-regulated anion channels that sense ionic strength. Cell 164: 499-511, 2016.

12. Trothe J, Ritzmann D, Lang V, Scholz P, Pul Ü, Kaufmann R, Buerger $\mathrm{C}$ and Ertongur-Fauth T: Hypotonic stress response of human keratinocytes involves LRRC8A as component of volume-regulated anion channels. Exp Dermatol 27: 1352-1360, 2018.

13. Pedersen SF, Okada Y and Nilius B: Biophysics and physiology of the volume-regulated anion channel (VRAC)/Volume-sensitive outwardly rectifying anion channel (VSOR). Pflugers Arch 468 371-383, 2016.

14. Kunzelmann K: Ion channels in regulated cell death. Cell Mol Life Sci 73: 2387-2403, 2016

15. Xu B, Jin X, Min L, Li Q, Deng L, Wu H, Lin G, Chen L, Zhang H, Li C, et al: Chloride channel-3 promotes tumor metastasis by regulating membrane ruffling and is associated with poor survival. Oncotarget 6: 2434-2450, 2015.

16. Sawada A, Takihara Y, Kim JY, Matsuda-Hashii Y, Tokimasa S, Fujisaki H, Kubota K, Endo H, Onodera T, Ohta H, et al: A congenital mutation of the novel gene LRRC8 causes agammaglobulinemia in humans. J Clin Invest 112: 1707-1713, 2003.

17. Choi H, Ettinger N, Rohrbough J, Dikalova A, Nguyen HN and Lamb FS: LRRC8A channels support TNF $\alpha$-induced superoxide production by Nox1 which is required for receptor endocytosis. Free Radic Biol Med 101: 413-423, 2016.

18. Rubino S, Bach MD, Schober AL, Lambert IH and Mongin AA: Downregulation of leucine-rich repeat-containing 8A limits proliferation and increases sensitivity of glioblastoma to temozolomide and carmustine. Front Oncol 8: 142, 2018.
19. Zhang H, Deng Z, Zhang D, Li H, Zhang L, Niu J, Zuo W, Fu R, Fan L, Ye JH and She J: High expression of leucinerich repeatcontaining 8A is indicative of a worse outcome of colon cancer patients by enhancing cancer cell growth and metastasis. Oncol Rep 40: 1275-1286, 2018

20. Sorensen BH, Nielsen D, Thorsteinsdottir UA, Hoffmann EK and Lambert IH: Downregulation of LRRC8A protects human ovarian and alveolar carcinoma cells against cisplatin-induced expression of p53, MDM2, p21Waf1/Cip1, and caspase-9/-3 activation. Am J Physiol Cell Physioly 310: C857-C873, 2016.

21. Sorensen BH, Dam CS, Sturup S and Lambert IH: Dual role of LRRC8A-containing transporters on cisplatin resistance in human ovarian cancer cells. J Inorg Biochem 160: 287-295, 2016.

22. Liu C, Zhang Y, Zhang K, Bian C, Zhao Y and Zhang J: Expression of estrogen receptors, androgen receptor and steroid receptor coactivator-3 is negatively correlated to the differentiation of astrocytic tumors. Cancer Epidemiol 38: 291-297, 2014.

23. Livak KJ and Schmittgen TD: Analysis of relative gene expression data using real-time quantitative PCR and the 2(-Delta Delta C(T)) method. Methods 25: 402-408, 2001.

24. Mirabello L, Troisi RJ and Savage SA: Osteosarcoma incidence and survival rates from 1973 to 2004: Data from the Surveillance, Epidemiology, and End Results Program. Cancer 115: 1531-1543, 2009.

25. Karpik M and Reszeć J: Low grade chondrosarcoma-epidemiology, diagnosis, treatment. Ortop Traumatol Rehabil 20: 65-70, 2018.

26. Estrada-Villaseñor EG, Flores-Carmona JF, Delgado-Cedillo EA and Rico-Martínez G: Bone tumor frequency in adults and elderly. Acta Ortop Mex 22: 356-360, 2008 (In Spanish).

27. Konishi T, Shiozaki A, Kosuga T, Kudou M, Shoda K, Arita T, Konishi H, Komatsu S, Kubota T, Fujiwara H, et al: LRRC8A expression influences growth of esophageal squamous cell carcinoma. Am J Pathol 189: 1973-1985, 2019.

28. Lu J, Xu F and Zhang J: Inhibition of angiotensin II-induced cerebrovascular smooth muscle cell proliferation by LRRC8A downregulation through suppressing PI3K/AKT activation. Hum Cell 32: 316-325, 2019.

29. Platt CD, Chou J, Houlihan P, Badran YR, Kumar L, Bainter W, Poliani PL, Perez CJ, Dent SYR, Clapham DE, et al: Leucine-rich repeat containing $8 \mathrm{~A}$

30. LRRC8A)-dependent volume-regulated anion channel activity is dispensable for T-cell development and function. J Allergy Clin Immunol 140: 1651-1659.e1, 2017.

This work is licensed under a Creative Commons Attribution-NonCommercial-NoDerivatives 4.0 International (CC BY-NC-ND 4.0) License. 